\title{
SHORT COMMUNICATION Food images engage subliminal motivation to seek food
}

\author{
H Ziauddeen', ${ }^{1,2}$, N Subramaniam ${ }^{1}$, R Gaillard ${ }^{3}$, LK Burke ${ }^{2}$, IS Farooqi ${ }^{2}$ and PC Fletcher ${ }^{1}$
}

Human eating behaviour is motivated and shaped by a complex interaction of internal drives such as hunger, external influences such as environmental cues and the sensory properties of food itself. Thus, as is demonstrated by the example of sensory-specific satiety (SSS), hunger may be reduced but particular foods (for example, desserts) retain their attraction and their ability to prompt consumption. In considering consumption, and overconsumption, it is therefore important to understand the interaction between internal and external drives to eat. Using grip force as a measure of motivation, we examined this interaction using an SSS manipulation. Critically, we sought to determine whether food stimuli would exert their influence even when they were subliminally presented (and thus not accessible to consciousness), and whether this unconscious influence would be flexibly updated in response to changes in food reward value with satiety. Demonstrating that the SSS effect remains when external stimuli are not consciously perceived, our data highlight the importance of even the most subtle, fleeting and even subliminal external events in shaping our motivation towards food.

International Journal of Obesity (2012) 36, 1245 - 1247; doi:10.1038/ijo.2011.239; published online 6 December 2011

Keywords: sensory-specific satiety; subliminal motivation; incentive force; food reward

\section{INTRODUCTION}

A reward's value influences the effort that we will expend to acquire it. In our environment, powerfully motivating food rewards are plentiful. A crucial determinant of the value of a food is the individual's current metabolic state. ${ }^{1}$ Hunger can make a food more appealing and desirable whereas satiety reduces this desire. However, as is well known, this effect of satiety can be sensory-specific, such that a food with different sensory properties, for example a dessert after a heavy main course, may still be attractive and desirable ${ }^{2}$ and indeed this may encourage greater consumption in environments where a variety of foods are available over a period of time. ${ }^{3}$ This phenomenon of sensoryspecific satiety (SSS) remains intact in amnesic patients. Such individuals may eat multiple meals owing to their memory deficit but while their desire to consume may be unaffected by recent meals, their preference is, even though they may have no explicit memory for what they previously consumed. ${ }^{4}$ This observation raises the intriguing possibility that an explicit awareness of the current value of a food is not necessary to drive and shape behaviour.

In considering unconscious motivators of food consumption two important questions arise. First, given the evidence that with rewarding non-food stimuli (for example, money) motivational processes can operate outside conscious awareness $s^{5,6}$ and are sensitive to reward magnitude, ${ }^{7}$ do we find a similar effect of food stimuli outside conscious awareness? This therefore was our first experimental question. In addition, though unconscious processes are believed to be insensitive to changes in context and reward value, $^{8,9}$ the work on amnesic patients offers a clue that this may not be the case. Our second question therefore was whether the unconscious motivational effects of two food stimuli were sensitive to a change in value applied to only one of those stimuli. That is, does SSS modify unconscious motivation?

\section{MATERIALS AND METHODS}

A total of 24 healthy lean graduate students ( 12 men and 12 women, mean age $25.6 \pm 4.1$ years, mean body mass index $21.7 \pm 1.8 \mathrm{~kg} / \mathrm{m}^{2}$ ) were recruited from the University of Cambridge after obtaining informed consent. Participants' liking for the test foods was checked with a food preference questionnaire at screening. They were informed that the purpose of the study was to see how food preferences change as people eat.

Participants performed two identical blocks of an incentive force task (modified from ${ }^{7}$ ) in which they exerted effort to win two food rewards: pizzas (savoury) and cake (sweet). Each block comprised 150 trials. At the beginning of each trial, the prize at stake was displayed as a motivating stimulus on the computer screen, preceded and followed by a mask image. On control trials, neutral stimuli (for example, a tape dispenser) were shown. On half the trials, the prize was displayed for 200 milliseconds (conscious) and on the rest it was displayed for 33 milliseconds (subliminal) (See Figure 1). Prior piloting showed that perception was subliminal at this display duration. Different pictures of the prizes were used in the conscious and subliminal trials to minimise direct motor specification effects. ${ }^{10}$ Each block featured 25 conscious and 25 subliminal presentations of the three trial types (pizza, cake and control) in random order. On each trial, squeezing a handgrip could win points towards the prize at stake. Participants were told that the harder they squeezed the more points they won. They were instructed to squeeze as much as they felt like on any given trial (including control trials). A fluid level on the screen provided feedback on the effort. This was intended to optimise their engagement with the task but the maximum level achievable varied randomly across trials. Participants were informed that this feedback was intended only as a rough indicator of their grip force but that across trials, different forces would result in different fluid levels. The latter was to ensure that participants did not simply aim for a target level on each trial. Participants were instructed that they were playing for food prizes, some of which would be hidden, and at the end of each block one of their two food

\footnotetext{
1Department of Psychiatry, University of Cambridge, UK; ${ }^{2}$ University of Cambridge Metabolic Research Laboratories, Institute of Metabolic Science, Addenbrooke's Hospital, Cambridge, UK and ${ }^{3}$ Laboratoire de Physiopathologie des Maladies Psychiatriques, Centre de Psychiatrie et Neurosciences, Université Paris Descartes, Centre Hospitalier Sainte-Anne, Paris, France. Correspondence: Dr H Ziauddeen, Institute of Metabolic Science, Addenbrooke's Hospital, Box 289, Level 4, Cambridge, CB2 OQQ, UK. 
a Hunger and fullness ratings

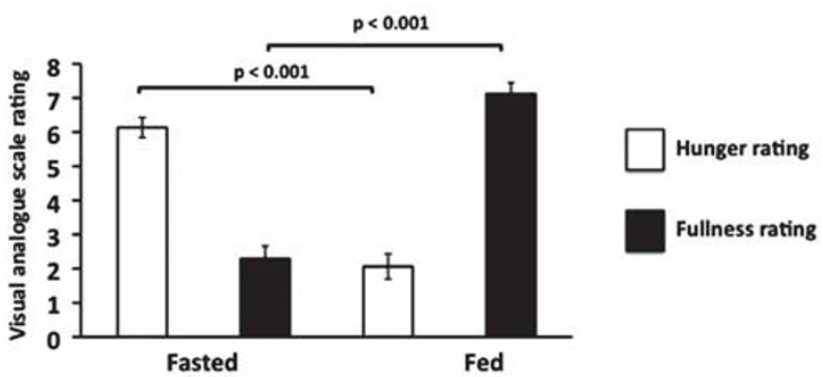

b Conscious condition
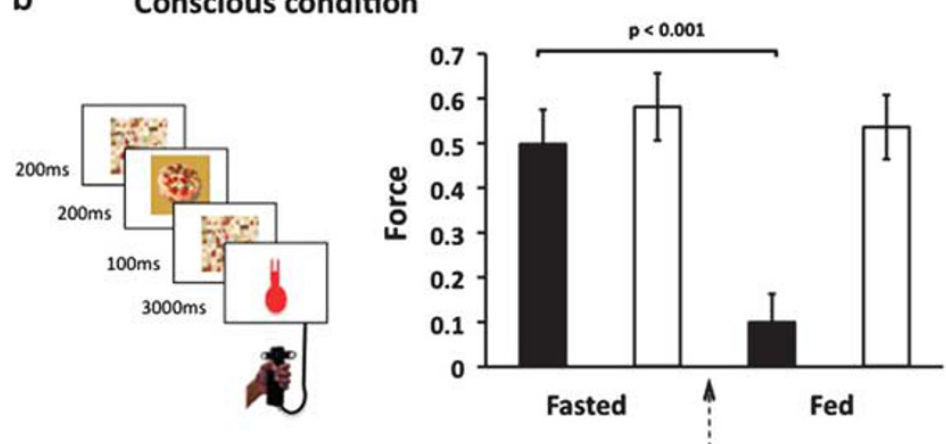

C Subliminal condition
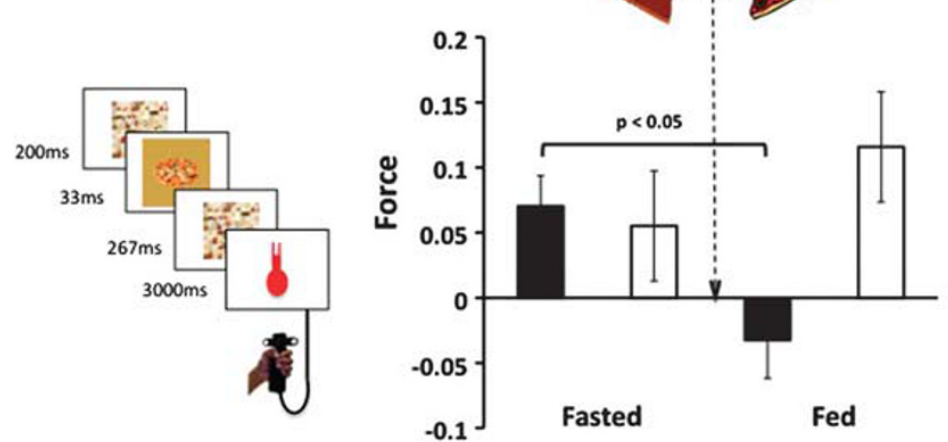

Figure 1. Specific satiety modulates motivational effort. (a) Change in hunger and fullness ratings with food consumption. (b, c) Participants exerted less for the food just consumed but still squeezed for the other food, regardless of awareness. Y-axis is the area under the curve normalised within subject and corrected for baseline changes.

caches would be randomly selected, with the amount depending on their performance. In fact, the allocated food and the amount (30\% of calculated daily calorie intake) were predetermined and counterbalanced across participants (13 of them won pizzas and 11 won cake). Both foods were modified to have identical macronutrient compositions (50\% carbohydrate, $30 \%$ fat and $20 \%$ protein). Following performance of the first block (fasted), participants consumed the allocated food before completing the second block (fed). Hunger and fullness ratings were collected on a 10-point visual analogue scale at the start of the session and after consumption of the food at the end of the first block. After the second block, participants were debriefed to determine whether they had been able to see the pictures on the subliminal trials and if so to estimate on what percentage of trials they had been able to do so. They were also asked whether they were aware of having varied their effort on the subliminal trials. A formal test of awareness was then performed using a forced choice discrimination task featuring 30 masked presentations of each of the subliminal pictures, each followed by two choices.

Stimulus-related motivation was measured as the total force exerted on the handgrip during each trial (the area under the force curve). Summary force measures were calculated for each trial type in both blocks. To reduce intersubject variability, the force measures were normalised within subjects according to the maximum of these measures. Corrections for nonspecific changes between the two blocks were made by subtracting responses on the control trials. We compared the effort exerted to win the food prizes before and after food consumption, using a linear mixed model with session (fed, fasted) and food (sated, non-sated) as fixed factors with a random intercept for subject, evaluating conscious and unconscious stimulus presentations separately.

One participant did not consume the food and was excluded from the analysis. One further participant was excluded from the analysis of subliminal trials because of superior performance on the forced choice discrimination task.

\section{RESULTS}

Hunger and fullness ratings changed significantly after the food had been consumed (Figure 1a); hunger ratings decreased (mean change -4.27 , s.d. $=1.91, t(22)=-15.357, P<0.001)$ and fullness 
Table 1. Parameter estimates from the linear mixed model analysis for conscious and subliminal trials

\begin{tabular}{llccc}
\hline Condition & Factor & Coefficient & s.e. & P-value \\
\hline Conscious $^{\mathrm{a}}$ & Session & -0.398 & 0.083 & $<0.001^{* *}$ \\
& Food & 0.083 & 0.067 & 0.214 \\
& Session $\times$ food & 0.353 & 0.094 & $<0.001^{* *}$ \\
Subliminal $^{\mathrm{b}}$ & Session & -0.104 & 0.052 & $0.045^{*}$ \\
& Food & -0.161 & 0.052 & 0.758 \\
& Session $\times$ food & 0.167 & 0.073 & $0.023^{*}$ \\
\hline *Significant at the 0.05 level. **Significant at the 0.001 level. $^{\mathrm{a}} \mathrm{N}=2$ 23. $^{\mathrm{b}} \mathrm{N}=22$.
\end{tabular}

ratings increased (mean change $=4.84$, s.d. $=2.58, t(22)=5.155$, $P<0.001)$. The analysis of the force data showed that as predicted, on conscious trials greater force was exerted for food, compared with neutral images. There was a significant SSS effect: during the second block, participants exerted less force to win the food they had just consumed, but were still working to win the other food (Figure 1b). Crucially, we found the same pattern of significant changes even on the subliminal trials (Figure 1c). The mixed model analysis revealed a significant effect of session and a session $\times$ food interaction for both the conscious (session: $P<0.001$, interaction: $P<0.001$ ) and subliminal trials (session: $P=0.045$, interaction: $P=0.023$ ). The parameter estimates from the mixed model are shown in Table 1. When debriefed after the second block, 19 participants reported no awareness of the subliminal pictures and 4 reported that they thought they had seen a pizza $5 \%$ of the time; all were unaware of having modified their exertion on these trials. Performance on the test of awareness was at chance levels and the $d$ prime index was not significantly different from zero (mean $=0.28$, s.e. $=0.17$, $P=0.109$ ). Thus, we can conclude that on these trials participants were not able to perceive the stimuli consciously, and therefore modulations of effort for the consumed food occurred outside conscious awareness.

\section{DISCUSSION}

We have demonstrated that subliminal motivation clearly occurs for food rewards. Moreover, it is sensitive to the current value of the food reward as determined by the individual's prevailing motivational state, the latter modulated by the experimental manipulation producing SSS. This sensory-specific unconscious devaluation may be mediated by the orbitofrontal cortex ${ }^{11}$ the neural substrate of SSS, through projections to the subcortical structures such as ventral striatum ${ }^{12}$ that subserve subliminal motivation. $^{7}$

These findings extend our understanding of unconscious influences on motivated behaviour by showing that they extend to food rewards, and by demonstrating that the current internal context (satiation with a specific food) can exert a valuedependent effect on unconscious motivation. Thus, internal metabolic state, the sensory properties of consumed foods and the availability of different foods in the environment, can interact to motivate behaviour outside of our conscious awareness.

\section{CONFLICT OF INTEREST}

The authors declare no conflict of interest.

\section{ACKNOWLEDGEMENTS}

We thank Rachel Woods for assistance with the study. HZ (RJAG/076), ISF (082390) and PCF (RNAG/031) are supported by the Wellcome Trust and the MRC Centre for Obesity and Related Metabolic Disease. This work was also supported by the National Institutes of Health Research Cambridge Biomedical Research Centre, the Bernard Wolfe Health Neuroscience Fund, GlaxoSmithKline and the Behavioural and Clinical Neuroscience Institute, University of Cambridge.

\section{REFERENCES}

1 Berridge KC. Motivation concepts in behavioral neuroscience. Physiol Behav 2004; 81: 179-209.

2 Rolls BJ, Rolls ET, Rowe EA, Sweeney K. Sensory specific satiety in man. Physiol Behav 1981; 27: $137-142$.

3 Rolls ET. Understanding the mechanisms of food intake and obesity. Obes Rev 2007; 8(Suppl 1): $67-72$.

4 Higgs S, Williamson AC, Rotshtein P, Humphreys GW. Sensory-specific satiety is intact in amnesics who eat multiple meals. Psychol Sci 2008; 19: 623-628.

5 Custers $\mathrm{R}$, Aarts $\mathrm{H}$. The unconscious will: how the pursuit of goals operates outside of conscious awareness. Science 2010; 329: 47-50.

6 Aarts $H$, Custers R, Marien $H$. Preparing and motivating behavior outside of awareness. Science 2008; 319: 1639.

7 Pessiglione M, Schmidt L, Draganski B, Kalisch R, Lau H, Dolan RJ et al. How the brain translates money into force: a neuroimaging study of subliminal motivation. Science 2007; 316: 904-906.

8 Dehaene S, Naccache L. Towards a cognitive neuroscience of consciousness: basic evidence and a workspace framework. Cognition 2001; 79: 1-37.

9 Merikle PM, Joordens S. Parallels between perception without attention and perception without awareness. Conscious Cogn 1997; 6: 219-236.

10 Neumann O. Direct parameter specification and the concept of perception. Psychol Res 1990; 52: 207-215.

11 Kringelbach ML, O'Doherty J, Rolls ET, Andrews C. Activation of the human orbitofrontal cortex to a liquid food stimulus is correlated with its subjective pleasantness. Cereb Cortex 2003; 13: $1064-1071$.

12 Fletcher PC, Napolitano A, Skeggs A, Miller SR, Delafont B, Cambridge VC et al. Distinct modulatory effects of satiety and sibutramine on brain responses to food images in humans: a double dissociation across hypothalamus, amygdala, and ventral striatum. J Neurosci 2010; 30: 14346-14355. 